\title{
stockage de chaleur dans les excavations à ciel ouvert ou souterraines
}

\author{
par \\ A. Montjoie \\ Université de Liège
}

RESUME - Ies excavations à ciel ouvert ou souterraines offrent d'intéressantes nossikilités de stockace de chaleur principalement sous forme d'eau chaude. Un inventaire complet de ces potentialités a été réalisé en Relgique. Il a conduit à retenir 122 carrières et une dizaine de puits de mines. Des tests expérimentaux ont été envisagés et sont décrits dans la présente communication.

Les carrières posent de nombreux problèmes du point de vue environnement aussi bien lors de leur exploitation que pour leur réaménagement ultérieur. $\AA$ ce sujet le stockage de chaleur, principalement sous forme d'eaux chaudes, peut constituer une utilisation intéressante. Si actuellement, on s'occupe uniquement des possibilités offertes par les carrières existantes, il n'est pas exclu de concevoir dans le futur une conduite des excavations favorisant leur utilisation dans ce domaine.

Le stockage de chaleur a pour but de modeler l'offre sur la demande. La première est souvent rigide, dépendant des activités industrielles susceptibles de fournir des calories généralement sous forme d'eau chaude ou encore des conditions météorologiques notamment dans le cas de la récupération de la chaleur solaire. La seconde fluctue fortement suivant la température extérieure, donc des saisons, et 1'horaire des activités humaines.

Dans le cas d'activités industrielles ou de chauffage urbain, l'ordre de grandeur des volumes minima à stocker est de $10.000 \mathrm{~m}^{3}$ si l'on souhaite réaliser une modulation journalière et de 1.000 .000 de $\mathrm{m}^{3}$ pour une modulation saisonnière.

Si le premier stockage peut être réalisé artificiellement sans trop de difficulté et même, dans le cas de chauffage urbain, être obtenu par l'inertie thermique des conduites et des immeubles, le deuxième présente un coût élevé et il y a lieu de rechercher des sites ou des structures naturelles favorables : carrières à ciel ouvert, carrières souterraines, puits et mines, nappes aquifères.

Dans le cadre du programme national belge R.D. Energie (Service Programmation de la Politique Scientifique), les Laboratoires de Géologie générale et appliquée de I'Université de Liège ont été chargés d'étudier les sites favorables pour l'expérimentation et l'implantation de système de stockage de chaleur.

Dans une première phase, on a réalisé $1^{\prime}$ inventaire des sites géologiques permettant l'application des techniques de stockage de chaleur sur base des méthodes les plus prometteuses(1). En fonction de cet inventaire, une sélection de sites intéressants a été faite et des études plus détaillées ont été entreprises pour đéboucher sur des projets expérimentaux.

Plus de 4.000 sites ont été inventoriés comprenant des carrières à ciel ouvert, des carrières souterraines, des puits et mines.

(1) Le choix des méthodes les plus intéressantes ont été faites en collaboration avec l'équipe du Professeur BRYCH de la Faculté Polytechnique de Mons 


\section{CARRIERES A CIEL OUVERT.}

L'utilisation de ces structures dépend essentiellement du mode de stockage envisagé. Rares sont les carrières qui offrent une géométrie assez régulière et une imperméabilité suffisante pour être utilisées telles qu'elles avec un couvercle isolant en surface. Par contre, si elles sont noyées, on peut, suivant la méthode scandinave, les doter d'un volume de stockage sous forme de jupe plastique cylindrique, ancrée au fond et munie d'un couvercle mobile isolant (méthode scandinave), ou sous forme d'une cloche complètement étanche vers le haut. Le procédé nécessite une profondeur d'eau suffisante d'au moins $15 \mathrm{~m}$, si possible $30 \mathrm{~m}$. Pour de plus faibles hauteurs, on peut envisager le stockage en cellules horizontales juxtaposées et communiquant par chicanes verticales alternativement supérieures et inférieures de façon à créer une stratification eau chaude - eau froide horizontale.

Les 4.000 sites inventoriés ont été classés en 5 catégories suivant leur possibilité d'utilisation :

\section{Catégorie 1 :}

carrières de forte hauteur d'eau $(H=30 \mathrm{~m})$ dans lesquelles des cloches plastiques de grands volumes sont envisageables. Dans cette catégorie sont également reprises les carrières souterraines noyées.

\section{Catégorie 2 :}

carrières de faible hauteur d'eau $(\mathrm{H}=10-15 \mathrm{~m})$, de faible surface libre. Des jupes plastiques de plus faibles dimensions sont encore applicables. Les modules cellulaires sont particulièrement indiqués.

\section{Catégorie 3 :}

carrières de faible hauteur ( $\mathrm{H}=10-15 \mathrm{~m}$ ) et de grande surface libre. Ici aussi les cloches plastiques sont d'application mais en plus grand nombre. Les modules cellulaires semblent les plus avantageux.

\section{Catégorie 4 :}

carrières de très faible hauteur d'eau $(\mathrm{H}=10 \mathrm{~m})$ présentant des possibilités d'aménagement en vue d'augmenter la hauteur d'eau. Ces aménagements peuvent consister en une étanchéisation soit par tapis d'argile, soit par projection de béton ou encore par un revêtement hydrofuge ou toute autre technique adéquate. Des digues faites de matériaux prélevés dans la carrière permettrait également d'augmenter la hauteur d'eau; ces digues demandent généralement une étanchéisation.

\section{Catégorie 5 :}

carrières ne présentant aucun intérêt dans l'état actuel des techniques.
En fonction de ces catégories, 122 carrières ont été sélectionnées. Elles sont subdivisées suivant leur possibilité de stockage journalier $(10.000 \mathrm{~m} 3)$ ou de stockage saisonnier $(1.000 .000 \mathrm{~m} 3)$. Pour la Belgique, le nombre de carrières par catégorie est le suivant :

\begin{tabular}{|c|c|c|}
\hline & & saisonnières \\
\hline atégorie & $2:$ & journalières \\
\hline atégorie & $3:$ & $\begin{array}{l}\text { journalières } \\
\text { saisonnières }\end{array}$ \\
\hline
\end{tabular}

Chacune des 122 carrières sélectionnées a fait I'objet d'une fiche technique comprenant les points suivants :

- le code de la carrière qui indique : le numéro de la carte au 1/10.000ème ou elle est localisée, le numéro de la carrière sur cette carte, la catégorie de la carrière, le type de matériau rocheux, le volume de stockage envisageable.

- le nom de la carrière.

- les coordonnées Lambert.

- les caractéristiques géologiques et hydrogéologiques : nature lithologique du terrain, âge, structure du gisement, présence de failles ou fractures avec indication éventuelle de la nature du remplissage, présence de poches de dissolution, existence d'eau souterraine, données de perméabilité et de transmissivité, débits d'exhaure, etc.

- la géométrie de la carrière.

- les producteurs de chaleur existant dans les environs avec information de : leurs coordonnées, la distance par rapport à la carrière, la puissance en $\mathrm{MW}$, la température de l'eau en degrés centigrades.

- les consommateurs de chaleur situés à proximité :

- réseau de chauffage urbain avec les coordonnées, la distance, la puissance du réseau (MW), la densité du réseau (MN/km)

- les autres consommateurs éventuels avec leurs coordonnées et la distance par rapport à la carrière.

- des remarques éventuelles.

On constate une nette différence entre les parties Nord et Sud du pays. En Wallonie, les possibilités sont multiples et relativement réparties sur l'ensemble du territoire; par contre, en Flandre sur base des critères de sélection choisis, quelques sites seulement existent. 
Cette différence est essentiellement due à la géologie des deux régions. En effet, en Flandre, les terrains rencontrés sont soit des sables, soit des argiles. La structure est subhorizontale et la nappe phréatique peu profonde. Les carrières du Nord $n$ 'ont donc pas intérêt à augmenter la profondeur des fouilles en raison du risque de venues d'eau importantes, des changements lithologiques verticaux, des problèmes de stabilité de talus en terrains meubles.

Par contre, en Wallonie, la structure plissée du substratum et/ou les variations latérales de faciès ont obligé les carrières à augmenter la profondeur des excavations. Etant donné le faible nombre de carrières de catégorie 1 en Flandre, un certain nombre de carrières noyées sur de très faibles hauteurs d'eau ( 5 à $10 \mathrm{~m}$ ) ont été sélectionnées dans la partie Nord du pays en vue d'un stockage cellulaire.

La répartition par province se présente comme suit :

\section{Flandre occidentale et orientale.}

Il y a peu de carrières de type 1 à 4. Quelques sites de catégorie 2 et 3 journaliers et/ou saisonniers existent à l'Est. Par contre les carrières noyées sur des hauteurs d'eau comprises entre 5 et $10 \mathrm{~m}$ sont très nombreuses et permettent d'envisager un stockage par stratification horizontale.

\section{Province d'Anvers et du Limbourg.}

Les caractéristiques sont semblables à celles des Flandres avec cependant quelques carrières de catégorie 2 ou 3 dans le Nord-Est du Limbourg.

\section{Province du Hainaut.}

Il existe de nombreuses carrières de catégorie 1 et de possibilité de stockage saisonnier. II s'agit principalement des exploitations de calcaires de la région de Tournai et de carrières de la région de Quenast - Lessines. Les plus grandes carrières sont toujours en activité. Aux environs de Mons, d'anciennes exploitations souterraines de phosphate de chaux représentent des volumes permettant un stockage saisonnier.

\section{Province de Namur.}

Les carrières de catégorie 1 sont nombreuses et relativement bien dispersées sur l'ensemble du territoire. On observe également des carrières de catégorie 2 et 4.

\section{Province de Liège.}

Les carrières de catégorie 1 et de capacité de stockage journalier existent dans la partie Est de la province. A l'Ouest, il s'agit principalement de carrières aménageables de catégorie 4 .

\section{Province du Luxembourg.}

Quelques carrières de catégorie 1 et 2 existent dans la partie centrale de la province.

En ce qui concerne les projets de démonstration, deux sites ont été retenus : l'un près de Tournai, l'autre près de Mons et un troisième site a été envisagé en Flandre dans une carrière peu profonde.

Le site retenu près de Tournai correspond à une ancienne exploitation de calcaire tournaisien représentant un volume de $2.000 .000 \mathrm{~m} 3$ sous la nappe aquifère avec une hauteur d'eau atteignant $40 \mathrm{~m}$. Des producteurs importants de chaleur (cimenteries) existent dans un rayon de 2 à $5 \mathrm{~km}$ et les consommateurs potentiels sont constitués, outre ces industries, par les villes de Tournai et Antoing distantes de 2 à $3 \mathrm{~km}$. La perméabilité des terrains est relativement peu élevée et la géométrie de l'excavation permet d'envisager le stockage par jupe plastique ou par cloche, cette dernière étant entièrement immergée et par conséquent plus favorable au point de vue environnement.

En ce qui concerne le projet de démonstration trois dimensions de cloches ont été retenues soit :

\begin{tabular}{cccr} 
Cloche & $\begin{array}{c}\text { Hauteur } \\
(\mathrm{m})\end{array}$ & $\begin{array}{c}\text { Diamètre } \\
(\mathrm{m})\end{array}$ & \multicolumn{1}{c}{$\begin{array}{c}\text { Volume } \\
\left(\mathrm{m}^{3}\right)\end{array}$} \\
\hline & 35 & 20 & 11.000 \\
1 & 20 & 10 & 1.570 \\
3 & 10 & 5 & 195 \\
\hline
\end{tabular}

Ia cloche 1 représente l'unité de stockage en vraie grandeur applicable aux carrières de la catégorie 1.

La cloche 2 à celles des catégories 2 et 3.

La cloche 3 permettrait d'étudier les effets d'échelle et être utilisée dans de petites excavations.

Si l'on admet une perte de $10 \%$ de la capacité de stockage, $M$. SAROT de l'équipe de Mons a calculé que, pour un matériau ayant un $\lambda=0,025 \mathrm{Kcal} / \mathrm{h} \cdot \mathrm{m} .{ }^{\circ} \mathrm{C}$, 11 faut prévoir une épaisseur de $10 \mathrm{~cm}$; pour $\lambda=0,040$ $\mathrm{Kcal} / \mathrm{h} \cdot \mathrm{m} .{ }^{\circ} \mathrm{C}$, il faut $16 \mathrm{~cm}$ de matériaux isolants.

La résistance mécanique de la cloche doit pouvoir reprendre la poussée d'Archimède du matériau constitutif de la cloche (plus léger que l'eau) et la force de poussée de l'eau chaude moins dense que l'eau de la carrière, soit 


\begin{tabular}{|c|c|c|}
\hline Cloche & $\mathrm{c}=\underset{\mathrm{T} / \mathrm{m}}{10 \mathrm{~cm}}$ & $\begin{array}{c}\mathrm{c}=\underset{\mathrm{T} / \mathrm{m}}{16 \mathrm{~cm}} \\
\end{array}$ \\
\hline 1 & 10 & 13 \\
\hline 2 & 4 & 5,5 \\
\hline 3 & 1,6 & 2,3 \\
\hline
\end{tabular}

T/m : Tonnes par mètre linéaire circonférentiel.

Les ancrages ou tests devront reprendre cette poussée.

En ce qui concerne les carrières souterraines, un test expérimental a été envisagé dans une ancienne exploitation souterraine de craie, à proximité de Mons.

De nombreux producteurs d'eau chaude se situent à proximité et les agglomérations de Mons et de Quaregnon sont des consommateurs potentiels intéressants.

Le terrain contient une nappe aquifère importante présentant une porosité de fissures de 2 à 5 \% pouvant atteindre 10 à 15 ò dans les zones déconsolidées ou karstifiées.

Les essais de pompage réalisés à proximité indiquent des perméabilités de l'ordre de 1 à $10 \cdot 10^{-3} \mathrm{~m} / \mathrm{sec}$ dans les zones les plus perméables et 1 à $5 \cdot 10^{-4} \mathrm{~m} / \mathrm{sec}$ dans les secteurs plus compacts.

La géométrie de l'excavation se présente sous forme de chambres d'une hauteur de 4 à $10 \mathrm{~m}$ et de section carrée de l'ordre de $4 \mathrm{~m} \times 4 \mathrm{~m}$. Les piliers ont des dimensions équivalentes. Le volume total des vides est estimé à 3 millions de $\mathrm{m}^{3}$. En raison de la géométrie de l'excavation et des caractéristiques hydrogéologiques, la solution envisagée pour le stockage est l'aménagement de chaque chambre en cellule étanche, étanchéité réalisée par exemple par béton projeté sur treillis métallique boulonné au terrain. Les différentes cellules seraient raccordées de façon à fonctionner suivant un mode de stockage cellulaire.

Le coût élevé d'une telle mise en place a conduit à reporter l'expérimentation de ce site après avoir testé ce mode de stockage dans une carrière à ciel ouvert sous faible hauteur d'eau. Ce dernier test est prévu pour une carrière en Flandre et ne présente pas de problème particulier. Sa construction peut être réalisée à ciel ouvert dans le fond de la carrière après rabattement temporaire de la nappe.

\section{PUITS DE MINES.}

En ce qui concerne le stockage de chaleur dans les anciennes exploitations minières, on peut distinguer les puits proprement dits et les anciens travaux miniers. Ces derniers, qui présentent un volume extrêmement variable, ne permettent pas de garantir une étanchéité suffisante ni une pérénnité de la stabilité des excavationssuite aux modifications thermiques induites par le stockage. Seule une étude in situ approfondie permettrait d'étudier la fiabilité d'un tel système à long terme et les températures compatibles avec l'altération des terrains schisteux préalablement exploités. De plus, dans le Sud du pays, pratiquement toutes les exploitations minières ont fait l'objet de travaux jusqu'à très faible profondeur, voire en surface sur les versants de vallées, et les structures intéressantes sont extrêmement limitées. Il n'en est pas de même en Campine où la couverture de terrains secondaires et tertiaires est importante mais les mines y sont toujours en activité.

En ce qui concerne les puits proprement dits, leur étanchéité peut être réalisée aisément par la mise en place de revêtement en plastique. Malheureusement, la plupart ont été remblayés (obligation légale) en sorte que peu de sites sont actuellement disponibles. En Wallonie, 7 sièges, comprenant chacun 2 puits, sont encore accessibles; chacun d'eux a fait l'objet d'une fiche donnant : nor et numéro du puits, ses coordonnées, sa géométrie et son volume, les débits d'exhaure.

Le mode de stockage envisagé est :

- la fermeture des envoyages de façon à isoler le puits des anciens travaux miniers

- le déséquipement du puits

- l'étanchéisation de celui-ci soit naturelle soit par pose d'un revêtement plastique du même type que celui des cloches envisagées pour les carrières en surface.

Le volume ainsi isolé permettrait un stockace similaire à celui prévu dans les carrières mais avec une hauteur de plusieures centaines de mètres. De tels ouvrages seraient comparables au stockage envisagé par forage de très grand diamètre (turboforage mis au point par les Laboratoires de la Faculté Polytechnique de Mons Prof. BRYCH).

L'investissement important nécessité par l'équipement d'un puits de mine tel que prévu ci-dessous a conduit à envisager un projet de démonstration sur un puits existant de plus petite dimension. Or, dans le cadre de l'étude d'un projet de métro à Liège, 4 puits d'environ $30 \mathrm{~m}$ de profondeur ont été réalisés. Le puits 
Val-Benoit semble le plus favorable étant donné son accessibilité et la proximité de la centrale thermique universitaire. D'une profondeur de $34 \mathrm{~m}$, il est prolongé par une galerie de $12 \mathrm{~m}$ à son pied.

De plus, un réseau important de piézomètres a été établi pour étudier la nappe des graviers de Meuse et la nappe du bed rock.

Le puits traverse $3 \mathrm{~m}$ de limons et remblais, $7 \mathrm{~m}$ de graviers perméables et pénètre d'une vingtaine de mètres dans le substratum constitué de schistes et grès houillers avec quelques veines de charbon.

Les essais géomécaniques réalisés ont montré des résistances en compression simple variant en moyenne de $200 \mathrm{~kg} / \mathrm{cm}^{2}$ pour les schistes à $400 \mathrm{~kg} / \mathrm{cm}^{2}$ pour les schistes sableux et $900 \mathrm{~kg} / \mathrm{cm}^{2}$ pour les grès. Sous l'eau, les résistances moyennes tombent à $80 \mathrm{~kg} / \mathrm{cm}^{2}$ pour les schistes, $130 \mathrm{~kg} / \mathrm{cm}^{2}$ pour les schistes sableux et se maintiennent aux environs de $900 \mathrm{~kg} / \mathrm{cm}^{2}$ pour les grès. Les résistances moyennes en traction sont respectivement, pour les mêmes types de roches, de 30,35 et 100 $\mathrm{kg} / \mathrm{cm}^{2}$ pour les terrains secs, de 9,30 et $40 \mathrm{~kg} / \mathrm{cm}^{2}$ pour les terrains saturés. Les modules de déformation sur échantillons de laboratoire sont en moyenne de 50.000, 100.000 et $250.000 \mathrm{~kg} / \mathrm{cm}^{2}$ en terrains secs, de $30.000,70.000$ et $250.000 \mathrm{~kg} / \mathrm{cm}^{2}$ en terrains saturés tandis que les valeurs moyennes des modules d'élasticité sont respectivement de $85.000,160.000$ et $300.000 \mathrm{~kg} / \mathrm{cm}^{2}$ en terrains secs, 45.000 , 100.000 et $300.000 \mathrm{~kg} / \mathrm{cm}^{2}$ en terrains humides.

Les valeurs moyennes obtenues in situ sont :

\section{schis te}

- module de déformation

- module d'élasticité

$50.000 \mathrm{~kg} / \mathrm{cm}^{2}$ $80.000 \mathrm{~kg} / \mathrm{cm}^{2}$

schiste sableux

- module de déformation

- module d'élasticité

$60.000 \mathrm{~kg} / \mathrm{cm}^{2}$ $90.000 \mathrm{~kg} / \mathrm{cm}^{2}$

grès

- module de déformation

- module d'élasticité

$60.000 \mathrm{~kg} / \mathrm{cm}^{2}$ $100.000 \mathrm{~kg} / \mathrm{cm}^{2}$

En ce qui concerne les caractéristiques hydrogéologiques, la perméabilité est élevée dans les alluvions de l'ordre de 2 à $4 \cdot 10^{-3} \mathrm{~m} / \mathrm{sec}$, nettement réduite dans le bed rock avec des valeurs de $10^{-6}$ à $10^{-7}$ $\mathrm{m} / \mathrm{sec}$.

Les alluvions graveleuses de la Meuse, très perméables, sont isolées par un réseau de palplanches et le bed rock sous-jacent est peu perméable dans son ensemble sauf les quelques horizons gréseux. La surface piézométrique est subhorizontale indiquant un faible écoulement général dans le secteur.

On peut dans un premier stade envisager le stockage sans dispositif d'étanchéité. Le volume utilisable est de l'ordre de $850 \mathrm{~m}^{3}$. La zone libre entre la nappe et le terrain naturel permet de compenser la différence de densité due à l'eau chaude. En réalisant un éventuel forage à l'extrémité de la galerie mettant en communication la nappe alluviale avec le fond du puits, on pourrait exécuter un système de vases communicants, l'eau chaude injectée dans le puits refoulant l'eau froide dans la nappe alluviale via le forage de communication nappe alluviale - galerie et vice versa.

La production de chaleur pourrait Ere réalisée par une chaudière autonome d'une puissance de $2.10^{-6} \mathrm{~kg} /$ calorie $/ \mathrm{H}$ permettant le remplissage du puits en 40 heures environ.

Il semble cependant préférable d'utiliser l'eau chaude de la centrale thermique de l'Université de Liège au Val-Benolt. Elle est susceptible de fournir le volume de $850 \mathrm{~m}^{3}$ d'eau à $90^{\circ} \mathrm{C}$ dans un temps de 15 à 20 heures. L'eau récupérée pourrait être réinjectée dans le circuit de chauffage de l'Université. On testerait ainsi un système global : centrale thermique, conduites, stockage, refoulement.

L'essai du Val-BenoIt semble particulièrement intéressant car il s'agit d'un essai en grandeur nature en ce qui concerne les diamètres que l'on peut envisager ultérieurement dans un turboforage. D'autre part, la hauteur de $30 \mathrm{~m}$ permet une bonne étude correcte de la stratification de l'eau et du comportement thermique des terrains.

Le coût global de l'opération est estimé à 10.000.000 FB.

\section{CONCLUSION}

En conclusion, lorsqu'on songe aux réaménagements des sites, il semble que l'utilisation des carrières pour le stockage d'eau chaude mérite d'être envisagée principalement lorsqu'elles sont noyées avec une hauteur d'eau dépassant la dizaine de mètres. En ce qui concerne les puits de mines, leurs équipement pour le stockage d'eau chaude est relativement simple. Dans tous les cas, un critère très important 


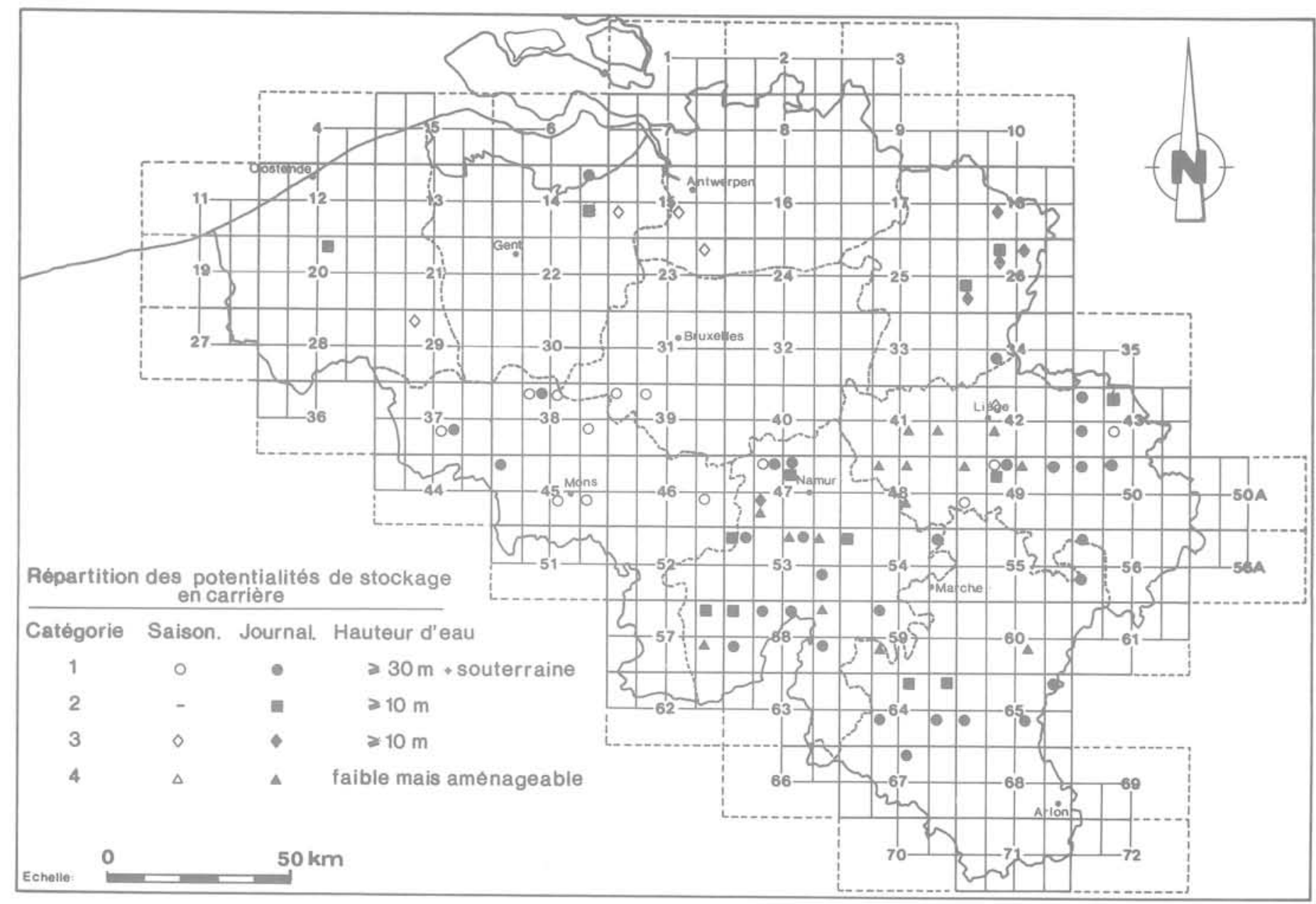

est la présence à proximité de producteurs et de consommateurs de chaleur. Les nappes aquifères présentent d'autres possibilités qui ont été également étudiées mais ne font pas l'objet de la présente note.

\author{
Laboratoires de Géologie \\ de l'Ingénieur et \\ d'Hydrogéologie
}

Faculté des Sciences appliquées de

l'Université de Liège.

\section{BIBLIOGRAPHIE SOMMAIRE}

Calembert L, Monjoie A \& Marchand L, (1979) Caractéristiques géologiques et hydrogéologiques des sites possibles cee stockage souterrain de chaleur. Colloque "Chauffage Urbain", 10 et 11 décembre 1979, Liège; pp. 204/P.1 $204 /$ P. 7.

Calembert $I_{\text {y }}$ Monjoie A \& Marchand L, (1980) Caractéristiques du sol intéressant les problèmes de production et de stockage de chaleur. Colloque "Energy savings in Buildings" 29 - 30 mai 1980, Namur; pp. 188 à 200.

Le texte qui précẽde est extrait d'un rapport du programme national $R-D$ Energie (Service du Premier Ministre -

Programmation de la Politique Scientifique - rue de la Science, 8, 1040 Bruxelles Belgique). Toute référence au présent texte doit comporter la mention des sources. La responsabilité scientifique du contenu de ce texte est assumée par ses auteurs. 


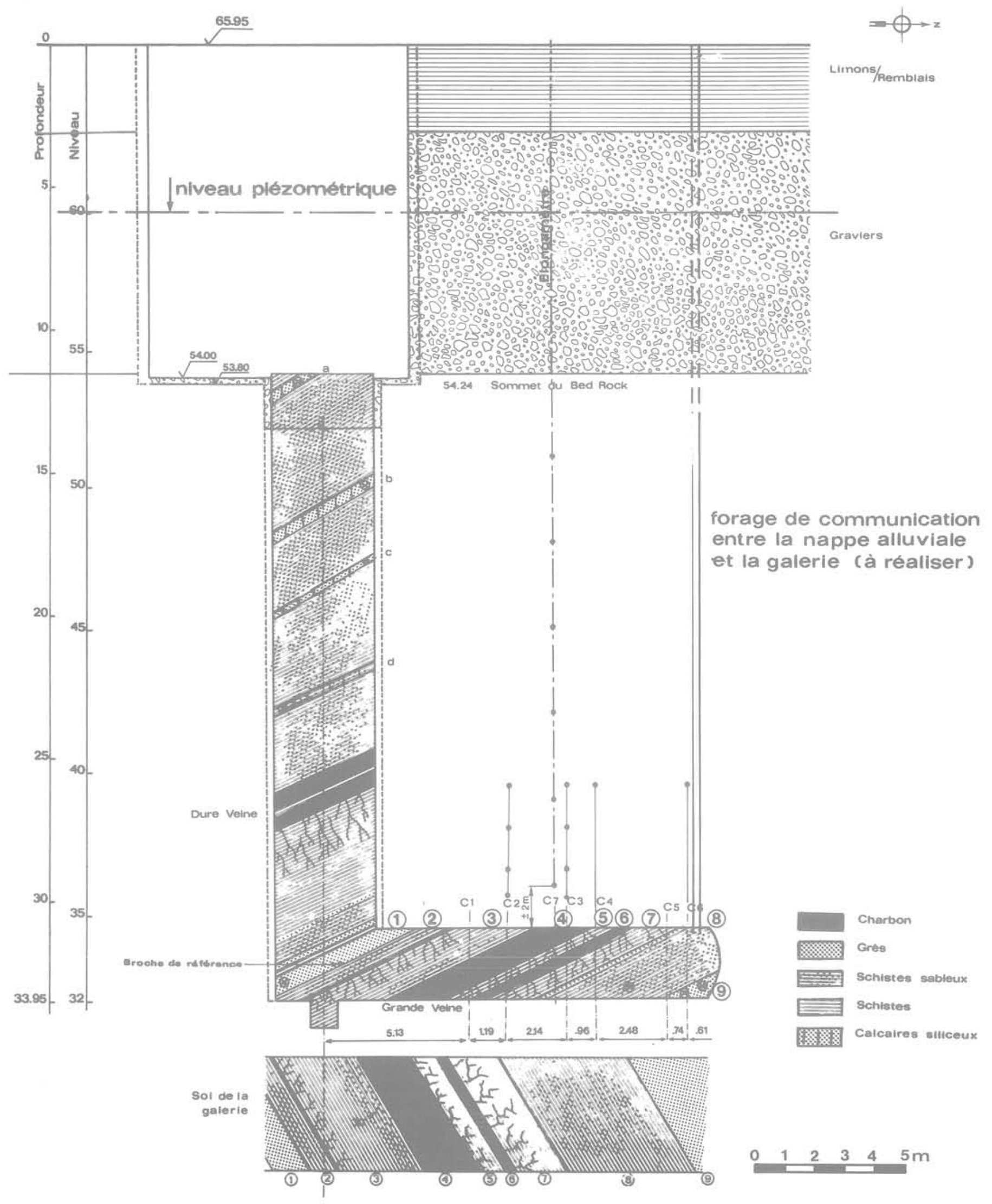

\section{Puits VAL BENOIT Coupe géologique par axe galerie}

